en cienciase

\section{Registro altitudinal inusual de Zorro Andino Pseudalopex culpaeus (Carnivora: Canidae) en el sureste de Ecuador}

\section{Leonardo Ordóñez-Delgado"* , Catherine Vitss', Ivonne González³ , DarwinValle ${ }^{4}$}

"Laboratorio de Ecología Tropical y Servicios Ecosistémicos - EcoSs Lab, Departamento de Ciencias Biológicas - Universidad Técnica Particular de Loja. CP: 11-01-608, Loja, Ecuador ¿Copalinga Ecolodge, Vía al Parque Nacional Podocarpus km 3. Zamora, Zamora Chinchipe Ecuador

¿Universidad Técnica Particular de Loja, Departamento de Ciencias Biológicas, Loja, Ecuador 4Programa de Maestría en Biología de la Conservación y Ecología Tropical. Departamento de Ciencias Biológicas - Universidad Técnica Particular de Loja.

*Autor para correspondencia/Corresponding autor; e-mail: lyordonez2@utpledu.ec

\section{Unusual altitudinal record of Culpeo Pseudalopex culpaeus (Carnivora: Canidae) in southeastern Ecuador}

\section{Abstract}

We report an unusual altitudinal record of the Culpeo Pseudalopex culpaeus (Carnivora: Canidae) registered by trap cameras at $1353 \mathrm{~m}$ in the southeastern Andean foothills a record documented for this species in the country and is the first report for a region where its presence was not previously evidenced. As reference associated information we present the list of mammals from small to medium size registered in this locality.

Key words: Canidae, New Distribution, Andean Foothills, Podocarpus National Park.

\section{Resumen}

Reportamos un registro altitudinal inusual de Zorro Andino Pseudalopex culpaeus (Carnivora: Canidae) obtenido con cámaras trampa a 1353m en el piedemonte suroriental andino, Copalinga Ecolodge, provincia de Zamora Chinchipe, Ecuador. Este constituye el registro altitudinal más bajo de esta especie en el país y el primer reporte para una región en la que no existía evidencia previa de su presencia. Como información de referencia asociada, presentamos el listado de los mamíferos de pequeño a mediano tamaño registrados en esta localidad.

Palabras clave: Canidae, Distribución, Piedemonte Andino, Parque Nacional Podocarpus.
El género Pseudalopex se considera endémico para el Neotrópico y comprende un conjunto de seis especies de cánidos sudamericanos de tamaño pequeño a mediano [1]. Dos de estas especies se encuentran en Ecuador: el Zorro Andino Pseudalopex culpaeus y el Zorro de Sechura $P$. sechurae[2].

El Zorro Andino posee una distribución amplia que abarca principalmente ecosistemas altoandinos desde el sur de Colombia hasta Argentina [2,3]. Según la Unión Internacional para la Conservación de la Naturaleza UICN, esta especie se ubica en la categoría de Preocupación Menor (LC) a nivel global [4]. Sin embargo, la especie se considera como Vulnerable (VU) en Ecuador [5], principalmente debido a la pérdida y fragmentación del hábitat que ocupa, y a las presiones de cacería que enfrenta [5]. Se ha estimado que al menos el 58\% del área de distribución histórica de la especie se encuentra disturbada [2].El Zorro Andino habita en Ecuador los bosques templados y páramos andinos, entre los 2600 y $4810 \mathrm{~m}$ de altitud, con pocos registros a $1660 \mathrm{~m}$ en el valle del rí Guayllabamba, provincia de Pichincha; y en el sector de Guarumales, provincia del Azuay [2]. En esta publicación presentamos un registro inusual de Zorro Andino en la zona de amortiguamiento norte del Parque Nacional Podocarpus (PNP), en el piedemonte suroriental de los Andes.

Estos registros fueron logrados por medio de cámaras trampa, las mismas se colocaron de manera esporádica (sin un patrón definido), entre septiembre del 2011 y julio del 2016 a altitudes de entre 950 y 1450m en los senderos de Copalinga Ecolodge, cantón Zamora, provincia de Zamora Chinchipe. El sector corresponde a un bosque siempreverde montano bajo (vegetación de transición entre el bosque húmedo de tierras bajas y el bosque montano) [6]. Los arboles de la zona llegan a medir entre 30 y $40 \mathrm{~m}$ de altura, especies características del sitio son Metteniusa tessmanniana (Metteniusaceae), Terminalia oblonga, Terminalia amazonia (Combretaceae), Guarea guidonia (Meliaceae), Oenocarpus bataua y Ceroxylon amazonicum (Aracaceae) [6]. Protium sp (Bursaraceae) localmente conocida como copal e Inga sp. (Fabaceae) son las especies de árboles más comunes en el sitio e inspiraron el nombre de la reserva [7]. El clima predominante en esta región se define como subtropical, con temperaturas anuales que fluctúan entre los 18 y $21^{\circ} \mathrm{C}$ y régimen pluviométrico súper húmedo, con más de 2000mm de precipitación anual, sin estación seca a lo largo del año[8]

Un individuo adulto de Zorro Andino fue grabado en video por 20 segundos a las $22 \mathrm{~h}: 34$ altitud $\left(-4,087^{\circ} \mathrm{S},-78,967^{\circ} \mathrm{O}\right.$, WGS84). El video fue logrado con una cámara trampa Bushnell Trophy Cam HD ubicada a $1 \mathrm{~m}$ de altura, sin uso de cebo como atrayente. Esta cámara se programó para activarse por movimiento y tomar videos de 20 segundos y fotografías, permaneció activa las 24 horas del día, entre el 23 de diciembre del 2013 y el 04 de febrero del 2014, sumando un total de 44 días de muestreo. La grabación de video ha sido depositada en el archivo audio visual del Museo de Colecciones Biológicas de la Universidad Técnica Particular de Loja con el código (MUTPL-RA01) y en el repositorio en línea Figshare[9].

La identificación de la especie se realizó en base a las características descritas por Tirira [2] y la revisión de material fotográfico de la especie publicado en el portal Mamíferos del Ecuador [3]. Si bien la grabación del video es en blanco y negro, características distintivas de la especie han permitido su identificación. El individuo grabado es un cánido de tamaño mediano, de cuerpo compacto, con pelaje tupido, la cola es abultada y de color más claro hacia la base y más oscura desde su parte media hasta la punta. En la nuca y la parte superior del dorso se evidencia una tonalidad más oscura que el vientre y la parte 
21 anances

osterior de los brazos y muslos, sus orejas se ven algo pequeñas, erguidas y de form triangular (Fig.1).

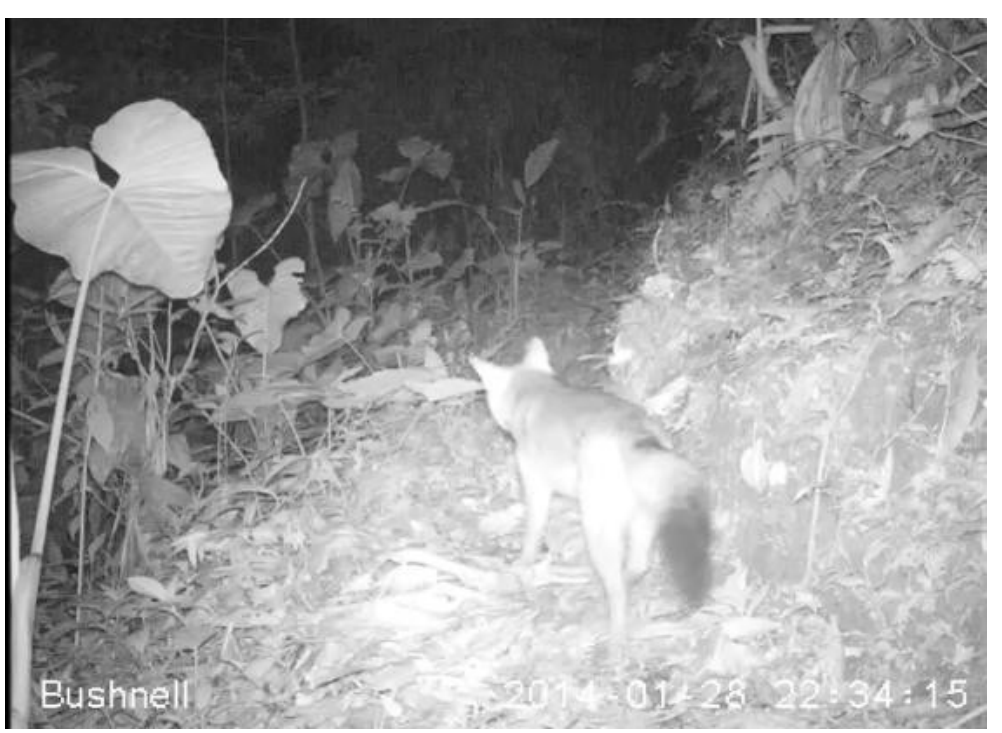

FIGURA 1. Fotograma extraído del video del Zorro Andino registrado en Copalinga Ecolodge en el piedemonte andino del sureste de Ecuador [6], el video completo puede ser observado en el enlace: https://doi.org/10.6084/ m9.figshare.4779745.v1

La cota altitudinal más baja de distribución del Zorro Andino para el país se ha establecido en $2600 \mathrm{~m}$ [2]. El registro aquí presentado se ubica a 1353m, es decir $1247 \mathrm{~m}$ bajo la cota de referencia previa (Fig. 2). Este registro es el de menor altitud para la especie en Ecuador y el único que se ubica en el suroriente del país. El lugar más cercano en donde se ha observado a la especie es Cajanuma, cerca de los páramos del Parque Nacional Podocarpus (L. Ordóñez - Delgado obs. pers.), a 2800 m de altitud y 22 km (en línea recta) al oeste de Copalinga Ecolodge. Este registro evidencia que la presencia de bosques continuos entre la parte baja, media y alta de esta región, los cuales son parte del Parque Nacional Podocarpus influyen positivamente en la presencia y movilidad de diversas especies faunísticas en el sector.

Además del registro del Zorro Andino, en los senderos de Copalinga, se han logrado fotografías demás de 19 especies de mamíferos, entre pequeños y grandes (Tabla1), de los cuales hasta la actualidad se han identificado 14 especies en base a Tirira [2]. De este listado, destaca adicionalmente el registro de Didelphis pernigra a $1035 \mathrm{~m}$, es deci $465 \mathrm{~m}$ bajo su rango de distribución altitudinal previamente conocido, el mismo que se establece entre 1500 y 4200m [2].

El Parque Nacional Podocarpus, es un área protegida de significativa importancia para la región sur, pero con poca información publicada sobre los mamíferos que alberga y protege, tema aún pendiente para fortalecimiento de su gestión. La toma de
TABLA 1. Listado de mamíferos registrados en Copalinga Ecolodge, Zamora, Zamora Chinchipe, sureste de Ecuador zona de a mortigumiento del Parque Nacional Podocarpus. Se presentan la altitud del registro en comparación a la altitud de referencia previamente conocida en el país en base a Tirira [2]

\begin{tabular}{|c|c|c|}
\hline $\begin{array}{l}\text { ORDEN } \\
\text { Familia } \\
\text { Especie }\end{array}$ & $\begin{array}{c}\text { Registro Copalinga } \\
\text { Ecolodge } \\
\text { (en m sobre el nivel del } \\
\text { mar) }\end{array}$ & $\begin{array}{c}\text { Distribución altitudinal } \\
\text { según Tirira [2] } \\
\text { (en m sobre el nivel del } \\
\text { mar) }\end{array}$ \\
\hline \multicolumn{3}{|l|}{$\begin{array}{l}\text { CARNIVORA } \\
\text { Canidae }\end{array}$} \\
\hline Psendalopex culpaeus & 1353 & 2600 a 4500 \\
\hline \multicolumn{3}{|l|}{ Felidae } \\
\hline Leopardus pardalis & 1065 & Oa 1500 \\
\hline Puma concolor & 1065 & 0 a 4500 \\
\hline Herpailurus yagouaroundi & 1185 & Oa 1800 \\
\hline \multicolumn{3}{|l|}{ Mustelidae } \\
\hline Eira barbara & $1015 / 1450$ & Oa 1550 \\
\hline \multicolumn{3}{|l|}{ Procyonidae } \\
\hline Bassaricyon alleni & 965 & 200 a 1600 \\
\hline Nasua nasua & 1035 & 200 a 1550 \\
\hline \multicolumn{3}{|l|}{$\begin{array}{l}\text { DIDELPHIMORPHIA } \\
\text { Didelphidae }\end{array}$} \\
\hline Didelphis marsupialis & 1035 & 0 a 2000 \\
\hline Didelphis pernigra & 1035 & 1500 a 3700 \\
\hline Metachirus nudicaudatus & 965 & 50 a 1200 \\
\hline \multicolumn{3}{|l|}{$\begin{array}{l}\text { LAGOMORPHA } \\
\text { Leporidae }\end{array}$} \\
\hline Sylvilagus brasiliensis & 965 & 0 a 4800 \\
\hline \multicolumn{3}{|l|}{$\begin{array}{c}\text { PILOSA } \\
\text { Myrmecophagidae }\end{array}$} \\
\hline Tamandua tetradactyla & 1015 & 200 a 1650 \\
\hline \multicolumn{3}{|l|}{$\begin{array}{l}\text { PRIMATES } \\
\text { Cebidae }\end{array}$} \\
\hline Cebus yuracus & 1015 & 0 a 2000 \\
\hline \multicolumn{3}{|l|}{$\begin{array}{l}\text { RODENTIA } \\
\text { Cuniculidae }\end{array}$} \\
\hline Cuniculus paca & 1015 & 0 a 2000 \\
\hline \multicolumn{3}{|l|}{ Dasyproctidae } \\
\hline Dasyprocta fuliginosa & 1015 & 200 a 1600 \\
\hline
\end{tabular}


decisiones acertadas en cuanto a la conservación de especies, debe fundamentarse en el conocimiento adecuado de él o los objetos de interés, siendo uno de los puntos de partida fundamentales para el efecto, el tener clara su distribución geográfica. El registro aquí presentado sobre Pseudalopex culpaeus, corrobora que aún existen vacíos de información sobre diversos elementos de la especie, incluida su distribución dentro de país, de ahí la urgente necesidad de incrementar investigaciones sobre la misma [5] que abarquen entre otros, temas de su ecología,movilidad, tamaños poblacionales y más elementos que aporten a su conservación

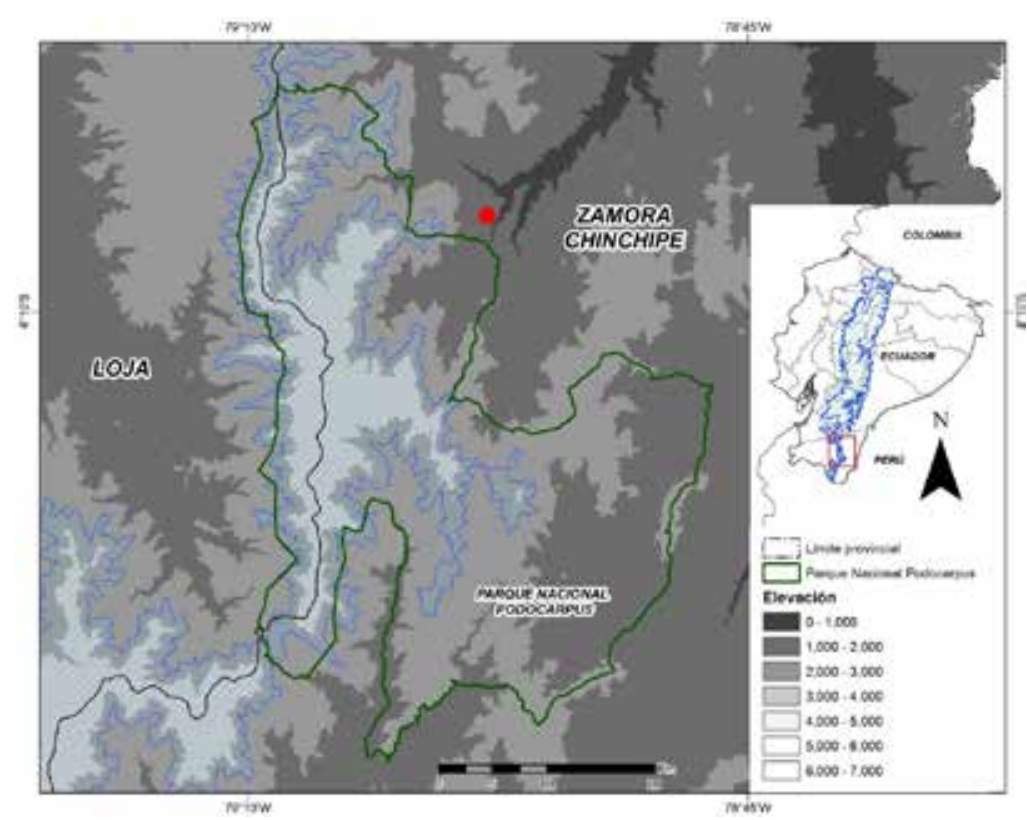

FIGURA 2. Mapa con la distribución potencial del Zorro Andino en Ecuador (área tramada) en base a Tirira [2] et punto rojo corresponde a la localidad de registro presentada en este documento.

\section{REFERENCIAS}

[1] Patterson, B. D., \& Costa, L. P. (Eds.). (2012). Bones, Clones, and Biomes: The history and geography of Recent Neotropical mammals. Chicago: University of ChicagoPress.

[2] Tirira, D. G. (2017). Guía de campo de los Mamiferos del Ecuador. Quito: Asociación Ecuatoriana de Mastozoología y Editorial Murciélago Blanco.

[3] Vallejo, A. F.,\& Moscoso, G. (2018). Pseudalopex culpaeus (Brito, J., Camacho, M.A., Romero, V., \& Vallejo, A. F. (Eds.)) En Mamiferos del Ecuador (Versión 2018.0) Museo de Zoología, Pontificia Universidad Católica del Ecuador. Recuperado el 30 de mayo de 2018 dehttps://bioweb.bio/faunaweb/mammaliaweb/FichaEspecie//Pseudalopex\%20culpaeus

[4] Lucherini, M. (2016). Lycalopex culpaeus. (IUCN) En The IUCN Red List of Threatened Species 2016. e. T6929A85324366. Recuperado el 23 de marzo de 2017 de http://dx.doi.org/10.2305/UCN.UK.2016-1.RLLS. T6929A85324366.en

[5] Zapata-Ríos, G., Tirira, D., Armijos, J., \& Cisneros, R. (2011). Lobo de páramo (Lycalopex culpaeus). (Tirira, D. (Ed.).) En Libro Rojo de los mamiferos del Ecuador. 2da. edición. Versión 1 (2011). Quito: Fundación Mamíferos y Conservación, Pontificia Universidad Católica del Ecuador y Ministerio del Ambiente del Ecuador.

[6] Balslev, H., \& øllgaard, B. (2002). Mapa de vegetación del sur de Ecuador. En Z. Aguirre, J. E. Madsen, E. Cotton \& H. Balslev (Eds.), Botánica Austroecuatoriana: Estudios sobre los Recursos Vegetales en las Provincias de EI Oro, Loja y Zamora-Chinchipe Quito: Ediciones Abya-Yala.

[7] Solano-Ugalde, A., Ordóñez-Delgado, L., Catherine, V, \& Freile, J. F. (2018) Breeding biology of Gray Tinamou (Tinamus tao) in southeastern Ecuador. The Wilson Journal of Ornithology. 130(2): 427-436. doi: http://wjoonline.org/doi/ $\mathrm{pdf} / 10.1676 / 16-229.1$

[8] Maldonado, N. (2002). Clima y vegetación de la región sur del Ecuador. En Z. Aguirre, J. E. Madsen, E. Cotton y H. Balslev (Eds.), Botánica Austroecuatoriana: Estudios sobre los Recursos Vegetales en las Provincias de El Oro, Loja y ZamoraChinchipe. Quito: Ediciones Abya Yala.

[9] Ordónez-Delgado, L. (2017).Video of Andean Fox in Copalinga, southeast of Ecuador. Figshare. Recuperado el 15 de abril del 2017 de https://doi.org/10.6084/m9.figshare.4779745.v

\section{CONTRIBUCIONES DE LOS AUTORES}

Leonardo Ordóñez-Delgado redactó el manuscrito, Catherine Vits realizó el levantamiento de datos de campo, Ivonne González trabajó las figuras y Darwin Valle consolidó el listado final de especies. Todos los autores efectuaron la revisión crítica del contenido intelectual del documento.

\section{AGRADECIMIENTOS}

Al Departamento de Ciencias Biológicas de la Universidad Técnica Particular de Loja por el respaldo institucional. A Diego Tirira por la revisión del video y confirmación en la identificación de la especie. A los revisores del documento por sus valiosos aportes para la consolidación del mismo. 